NASA Contractor Report 185167

AIAA-90-0344

\title{
An Interactive Grid Generation Procedure for Axial and Radial Flow Turbomachinery
}

Timothy A. Beach

Sverdrup Technology, Inc.

NASA Lewis Research Center Group

Cleveland, Ohio

November 1989

Prepared for

Lewis Research Center

Under Contract NAS3-25266

\section{N/SA \\ National Aeronautics and \\ Space Administration}

(NASA-CR-135167) AN INTERACTIVE GRIO

N90-13968

GENERATION PROCEDURE FUR AXIAL ANO RAOIAL

FLOW TURBOMACHINERY Final Report (Sverdrup

Technology) $13 \mathrm{~F}$

CSCL $\cap 90$ 


\title{
An Interactive Grid Generation Procedure
} for Axial and Radial Flow Turbomachinery

\author{
Timothy A. Beach \\ Sverdrup Technology, Inc. \\ NASA Lewis Research Center Group \\ Cleveland, Ohio
}

\begin{abstract}
A combination algebraic/elliptic technique is presented for the generation of three dimensional grids about turbomachinery blade rows for both axial and radial flow machinery. The technique is built around use of an advanced engineering workstation to construct several two dimensional grids interactivley on predetermined blade-to-blade surfaces. A three dimensional grid is generated by interpolating these surface grids onto an axisymmetric grid. On each blade-to- blade surface, a grid is created using algebraic techniques near the blade to control orthogonality within the boundary layer region and elliptic techniques in the mid-passage to achieve smoothness. The interactive definition of bezier curves as internal boundaries is the key to simple construction. This procedure lends itself well to zonal grid construction, an important example being the tip clearance region. Calculations done to date include a space shuttle main engine turbopump blade, a radial inflow turbine blade, and the first stator of the United Technologies Research Center large scale rotating rig. A finite volume Navier Stokes solver was used in each case.
\end{abstract}

\section{Introduction}

Generating grids which are smooth throughout and orthogonal at surfaces is fundamentally important when calculating viscous flows through turbomachinery blade rows. In addition to being quick, the process must be easy to use to be valuable for routine applications. To meet these goals, several existing techniques were combined in a unique manner to form a single interactive code to generate three dimensional grids for flow simulations through axial and radial flow turbomachinery.

Turbomachinery blade geometries often include combinations of high camber and high solidity, thick blades with blunt edges, and considerable twist. Combining these geometric constraints with those imposed by the solution procedure, for example, the pairing of grid points for periodic boundary conditions, leads to a major challenge in grid design. When applied to highly cambered blades, traditional elliptic techniques(1-3) produce smooth grids but often with insufficient control of boundary orthogonality. This is especially true with $\mathrm{H}$ type grids. Algebraic techniques (1,2,4-6) give better control at boundaries, although simple interpolation algorithms lead to grids which are not necessarily smooth. Thus the approach to grid generation described in this paper is a combination of algebraic and elliptic techniques. Strictly algebraic grids are maintained near the blade to control orthogonality in the boundary layer region, while elliptic techniques are employed to smooth out the mid-passage. The key to controlling orthogonality at the blades with simple linear interpolation techniques is the specification of a number of internal boundaries which have the desired shape. An interactive code called IGB was developed which allows this to be quickly accomplished on a graphics workstation.

Other features of the overall approach include generating a surface grid from blade, hub, and shroud coordinates and interpolating a number of interactively created blade-to-blade grids onto a single axisymmetric throughflow grid in order to produce a three dimensional grid. The procedure also lends itself well to zonal grid construction, an important example being the region between the blade tip and shroud. A sample of applications to date include a two stage (i.e., four blade row) Pratt and Whitney space shuttle main engine turbopump turbine, a NASA radial inflow turbine, and the first stator of the United Technologies Reasearch Center large scale rotating rig. These examples will be used to show both the range of applications and the quality of the grids generated. Also, results from the Navier Stokes solver are included for the radial turbine and the UTRC stator to illustrate some features of the grids produced by this code. Presently, the code runs on a Silicon Graphics IRIS 4D graphics workstation.

\section{Approach}

The IGB grid code consists of three main modules which are executed independently. The function of each will be briefly described for completeness. The interactive generation of the blade-toblade grid, which is the unique aspect of the present approach, will be discussed in some detail, since this step is the key to the overall success of the code.

The first step is to generate the bounding surface grid from the inputs, which include blade, hub, and shroud coordinates; grid dimensions; desired grid spacing at the leading and trailing edges; and several control parameters. At present this is a batch process with an option to redistribute the blade surface grid points interactively. If the blade 
coordinates do not include the leading and trailing edge, the blade is closed smoothly with circular arcs. Grid lines are packed from leading edge to trailing edge and from pressure side to suction side using the hyperbolic tangent function, and from hub to tip using the exponential function. Cubic splines $(7,8)$, continuous in first and second derivative, are used to model the blade surface and to interpolate the coordinates onto an axisymmetric throughflow grid, which is also created by this step. The nature of the inflow, outflow, and the wake regions can be treated in a number of ways specified by a few inputs. For example, inflow and outflow surfaces can be taken from a separate grid, as would be required for multi-blade row applications. The results of the surface grid generation step are a single axisymmetric throughflow grid and the surface grid for use in the blade-to-blade grid generation procedure. This information could also be generated using other surface geometry packages.

The second step of the IGB code is the interactive generation of a number of two dimensional grids, each of which lie on a blade-to-blade surface defined by the axisymmetric throughflow grid. This is done for the hub and shroud and several stations along the span. The number and location of the blade-to-blade grids done interactively is userspecified through an input file and will depend on the three dimensionality of the blade. It is a matter of judgement to provide enough surfaces to accurately account for three dimensionality, but not so many that radial smoothness is jeopardized by independently created two dimensional grids in close proximity. In general, radial continuity is maintained by treating each blade-to-blade surface in a consistant manner and by using spline interpolation to complete the three dimensional grid.

The key to the blade-to-blade grid generation step is the interactive definition of several internal boundaries, accomplished by placing a Bezier curve (7) between selected points on the grid boundary. The Bezier curve is ideal for this application because it is easily manipulated to provide the necessary internal boundary shape. Figure 1 shows the parametric equation as well as an example. The curve, which is defined by four control points, passes through the first and fourth control points, while the second and third determine its shape. The curve will always lie within the convex hull of the control points, and the slope at the endpoints will be that of the line joining an endpoint to an adjacent control point. When executing the code, it is simply a matter of choosing which grid lines to define as internal boundaries and then defining their shape by moving control points in a local polar coordinate system. The code has a convenient editing procedure whereby internal boundaries can be defined, deleted, or modified. In addition, a file containing a set of internal boundaries can be saved for later use. Figure 2 shows how the internal boundaries might look for an axial flow turbine application. Notice that they are shaped in such a way as to provide boundary orthogonality when the grid is filled by blade-to-blade interpolation.

The result of using linear interpolation (9) to construct the grid with the internal boundaries of Figure 2 is shown in Figure 3. The boundary intersections are orthogonal except at the leading and trailing edges. The mid-passage grid, however, is not smooth. The IGB code contains elliptic smoothing routines which can be applied to specified regions, which usually do not include the boundary layer region. Executing a series of elliptic solutions over various regions for just a few iterations each will usually result in a smooth grid. This can be done by entering the necessary information from the keyboard for each region, or by reading a file containing a series of smoothing inputs. Because the schedule of elliptic smoothing will be similar for most turbomachinery applications, the procedure becomes fairly simple with practice. The forcing functions used in the elliptic smoothing routines in this code have also been used by other authors(1-5). The formulation found most useful is from Soni (9), which is a modification of the method of Thomas and Middlecroft. Figure 4 shows the result of applying a series of elliptic smoothing steps to the grid in Figure 3.

It is neither required nor even desirable to create a grid on each blade-to-blade surface of a three dimensional grid in the above fashion, as this could lead to grids which are not radially smooth. Four or five surfaces, including the hub and shroud, are usually sufficient. Of course the number of surfaces depends on the three dimensionality of the blade. The empty blade-to-blade surfaces are filled using cubic splines to interpolate the interactively generated blade-to-blade grids onto the axisymmetric throughflow grid. This will result in a grid which is radially smooth if each blade-to-blade grid is produced in a consistent manner.

\section{Results}

The first example is a two-stage space shuttle main engine turbopump turbine designed by Pratt and Whitney. Figure 5 shows several passages in a perspective view of the blade and hub surface grids for the first rotor (second blade row). The plots shows 55 axial points, 25 radial points, and 31 blade-to-blade points, although the entire grid, including a turnaround duct, is $275 \times 25 \times 31$. The configuration can be seen clearly in Figure 6, where the axisymmetric grid is shown on top, and the full blade-to-blade grid for the fourth blade is on the bottom. The inset shows an enlargement of the trailing edge region. In the solution procedure, the effects of the neighboring blade rows are accounted for by body forces which are calculated from the detailed solutions of each blade row. An iterative procedure is used to allow the influence of each row to be felt by the entire machine. For more details 
on this approach to multi-blade row calculations, see references (10-11).

The second example, the first stator of the UTRC large scale rotating rig, will be examined more closely. Figure 7 shows a blade-to-blade grid surface plotted at mid-span of a $121 \times 41 \times 41$ grid. The insets show enlargements of the leading and trailing edge regions. These plots indicate the degree of orthogonality that is achieved at the blade surface without sacrificing overall grid smoothness. Figure 8 shows a further enlargement of a small region of the suction side of the blade just forward of the trailing edge, where the camber is high and orthogonality is most difficult to achieve. Velocity vectors from a Navier-Stokes solution are also plotted. The solution was obtained with an explicit finite volume code (12). The ability to keep several points on an orthogonal line within the boundary layer is critical in achieving accurate results using normal momentum boundary conditions and a simple zero order turbulence model or a wall function model. The trailing edge separation bubble and wake can easily be seen in Figure 9 , which is a plot of velocity vectors downstream of the trailing edge suction side. Alternate axial grid lines have been deleted for clarity. Clustering points in the wake is a requirement if losses are to be computed accurately. The IGB code allows for some flexibility when clustering points in the wake region. Initially, the grid lines are usually clustered along a guessed wake centerline, often an extrapolation of the mean camber line. The approximate location can be easily changed in another application of the IGB code if, after a preliminary flowfield calculation, it is found that the clustering missed the wake.

The flow through the clearance between the blade tip and casing can strongly influence the performance of a machine. Figure 10 shows the result of adapting this code to produce a grid block within the tip clearance region of a typical turbine blade. This is an $O$ type grid which collapses to a periodic boundary along the camber line. The clearance block matches exactly with the passage block so that no interpolation is required. No difficulties are expected in applying the finite volume method to this problem, although that work is not yet complete.

The radial inflow turbine shown in Figure 11 is the final example. The generation of grids for radial machines was a straightforward extension of the technique and produces the same quality results. One difference is that radial blades have relativly high curvature near mid-chord when compared to axial blades. This requires the ability to cluster the blade surface points at some point on the blade as well as at the leading and trailing edge. The same could also be required on a compressor for a passage shock impinging at a known blade location. Control of mid-chord clustering will also allow excessive skewness in the radial grid lines at the hub and casing to be minimized.

Figure 12 shows relative velocity vectors at grid points adjacent to the hub and blade suction surface. The solution was obtained with a finite volume code (12) and includes no tip clearance. Evidence of what is called the scraping vortex is seen near the tip where the flow migrating outward meets the flow moving radially inward. The blade sweeping through the casing boundary layer causes a vortex which is counter to the rotation of the passage vortex. Modelling the the tip clearance will reduce this effect. Investigation of this machine is the subject of a forthcoming paper (13).

\section{Conclusion}

Experience has revealed the IGB code to be a very useful tool in the computation of turbomachinery flowfields, A three dimensional grid for a new application can usually be accomplished in under three hours on a graphics workstation, and often takes less time. Minor changes after the initial work can be made quickly. With the proper attention to detail, the grid quality has been found to be very high, while the frustration level during the process is generally low. These features allow for more time and effort to be devoted to flow simulation and flow interpretation.

The IGB code is part of a trend in grid generation toward interactive codes. Although, the algebraic and elliptic techniques employed in it have been used for many years, it is the graphical interface that allows them to be applied easily and in new ways that can improve the quality of the resulting grids. There is still much room for improvement, however. The present code only begins to take advantage of the possibilities that interactive graphics has to offer grid generation.

\section{Acknowledgements}

The author wishes to thank Dr. Bharat Soni of Mississippi State University and Dr. Helen McConnehey of NASA Marshall Space Flight Center for much needed advice and assistance in the early stages of this effort. Thanks also to Jim Heidmann of NASA Lewis Research Center for the radial turbine calculation and to Dr. Kevin Kirtley of Sverdrup/LeRc for help in writing and debugging the grid code. The work was sponsored by the NASA Lewis Research Center under contract NAS3-25266 with Dr. John Adamczyk as moniter.

\section{References}

[1] Thompson, J.F., Numerical Grid Generation. North Holland, 1982.

[2] Thompson, J.F., Warsi, Z.U.A. and Mastin C.W. Numerical Grid Generation. North Holland, 1985. 
[3] Sorenson, R.L., and Steger, J.L. " Numerical Generation of Two-Dimensional Grids by the Use of Poisson Equations with Grid Control at Boundaries," Numerical Grid Generation Technique, Nasa Conference Publication 2166, 1980.

[4] Soni, B.K. "Two and Three Dimensional Grid Generation for Internal Flow Applications of Computational Fluid Dynamics," AIAA Paper No. 85-1526, 1985.

[5] Dorrell E.W., Jr. and Soni, B.K. "INGRD: Interactive Two-Dimensional Grid Generation," AEDC-TR-86-49, 1987.

[6] Mulac, R.A., "A Multistage Mesh Generator for Solving the Average Passage Equation System," NASA CR-179539, 1986.

[7] Faux, I.D. and PRATT, M.J. Computational Geometry for Design and Manufacture, John Wiley and Sons 1979.

[8] Akima, Hiroshi "A New Method of Interpolation and Smooth Curve Fitting Based on Local Procedures," Journalof the Association for Computing Machinery, Vol. 17, No. 4, October 1984.

[9] Soni, B.K., "GENIE: GENeration of Computational Geometry-Grids for Internal-External Flow Configurations", Numerical Grid Generation in Computational Fluid Mechanics '88, edited by Sengupta, et. al., Pineridge Press Limited, 1988.
[10] Adamczyk, J.J. "Model Equation for Simulating Flows in Multistage Turbomachinery," ASME Paper 85-GT-226, 1984 (NASA TM86869).

[11] Adamczyk, J.J., Mulac, R.A., and Celestina, M.L., "A Model for Closing the Inviscid Form of the Average Passage Equation System" ASME Paper 86-GT-227, 1986 (NASA TM87199).

[12] Adamczyk, J.J., Celestina, M.L., Beach, T.A., and Barnett, $M$. "Simulation of ThreeDimensional Viscous Flow within a Multistage Turbine," ASME Paper 89-GT-152.

[13] Heidman, J.D. and Beach, T.A., "An Analysis of the Viscous Flow Through a Compact Radial Turbine by the Average Passage Approach", Submitted to the 35th International Gas Turbine and Aero Engine Congress and Exposition, Brussells, Belgium, 1990.

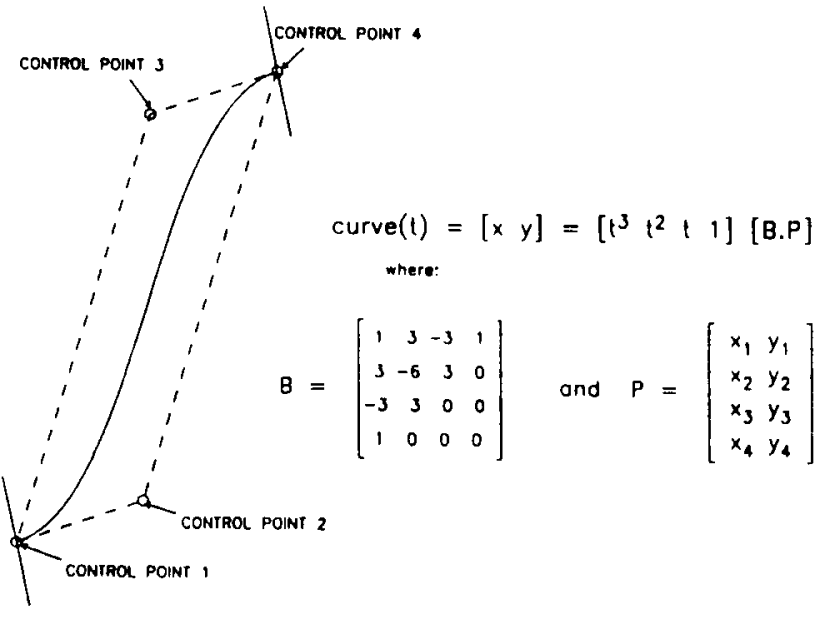

Figure 1. Parametric equation for a two dimensional Bezier curve with an example. 


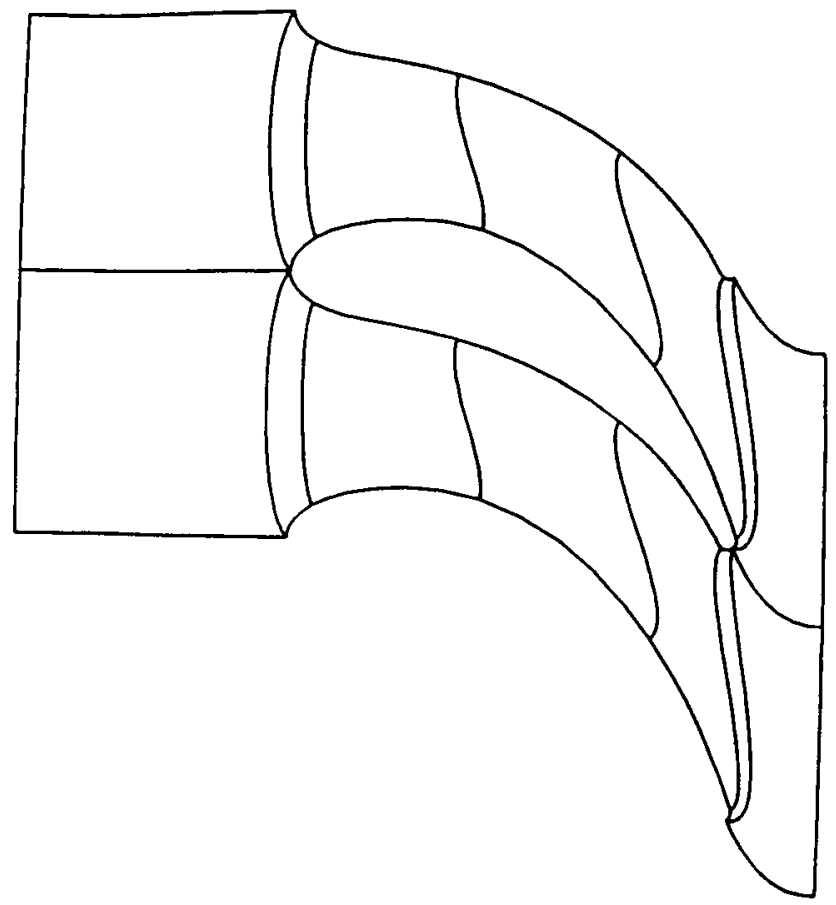

Figure 2. Internal boundaries for an axial turbine blade application. Bezier curves are shaped interactively so that they are orthogonal at the blade surface, except at the leading and trailing edges.

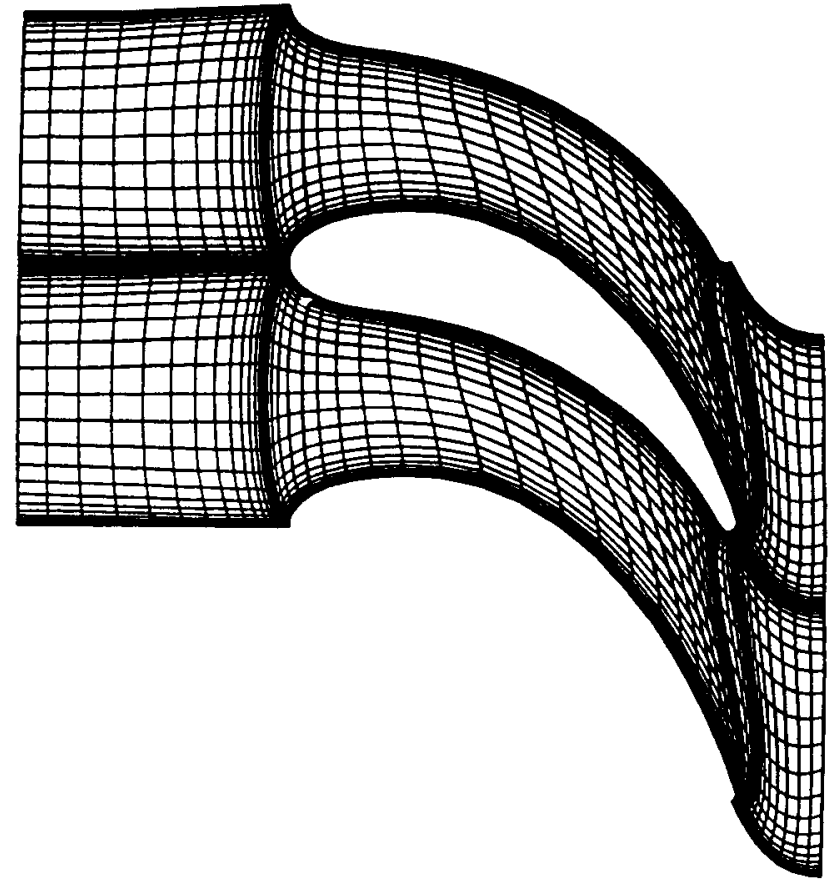

Figure 3. An algebraic grid produced by linear interpolation between the internal boundaries shown in Figure 2. Orthogonality is maintained at the blade surface, except at the leading and trailing edges. 


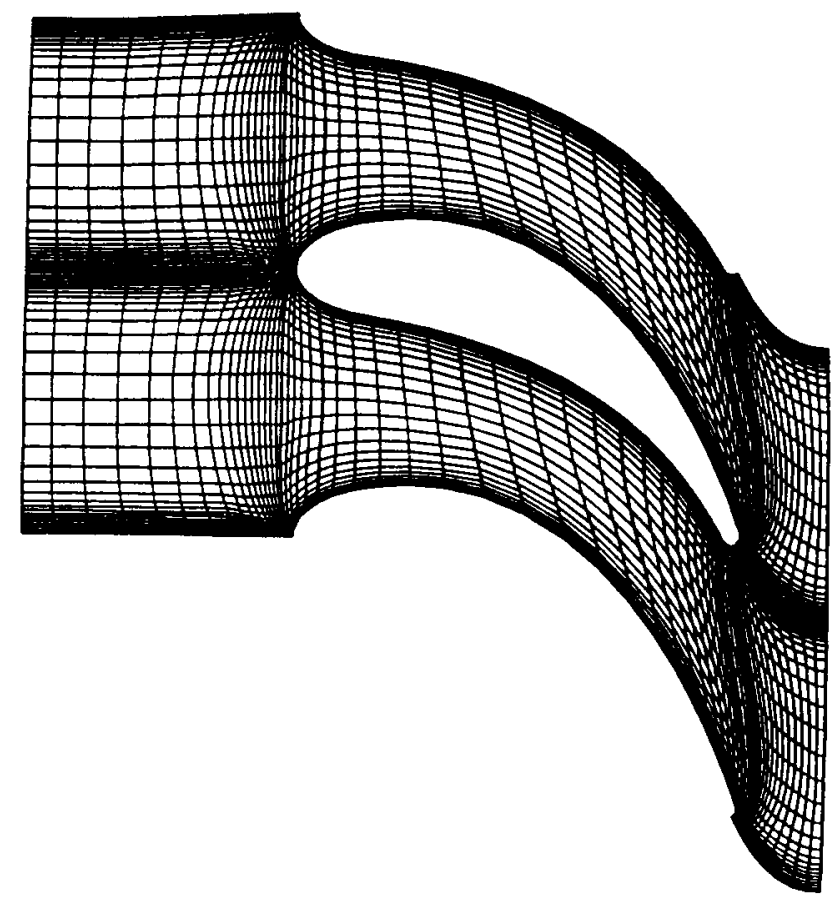

Figure 4. A final grid after elliptic smoothing. Since the algebraic grid near the blade has not been altered by the smoothing, orthogonality is maintained in that region.

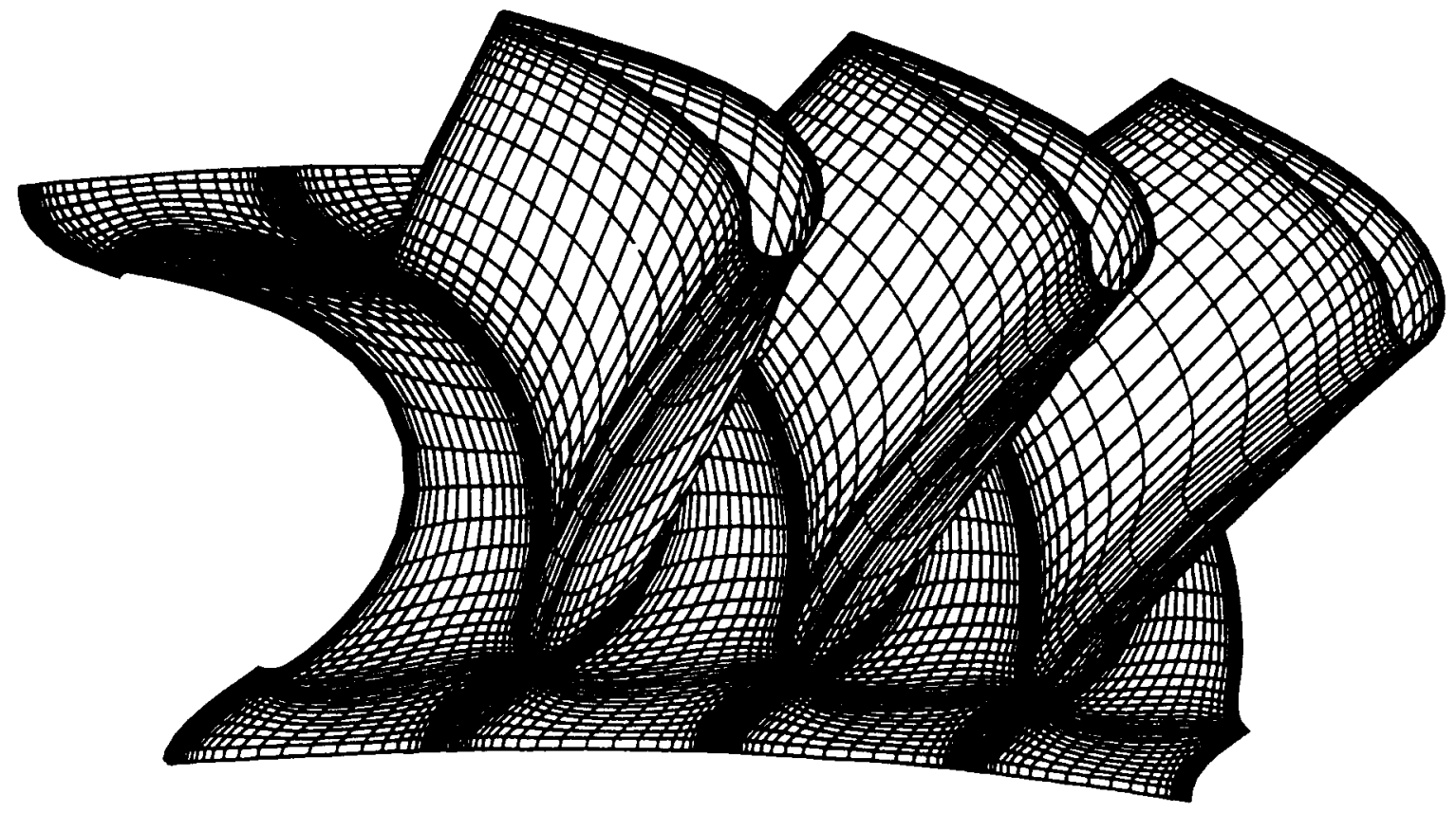

Figure 5. A perspective view of the first rotor (second bladerow) of the Pratt and Whitney space shuttle main engine turbopump turbine. There are 55 axial points, 25 radial points and 31 blade-to-blade points per passage. 


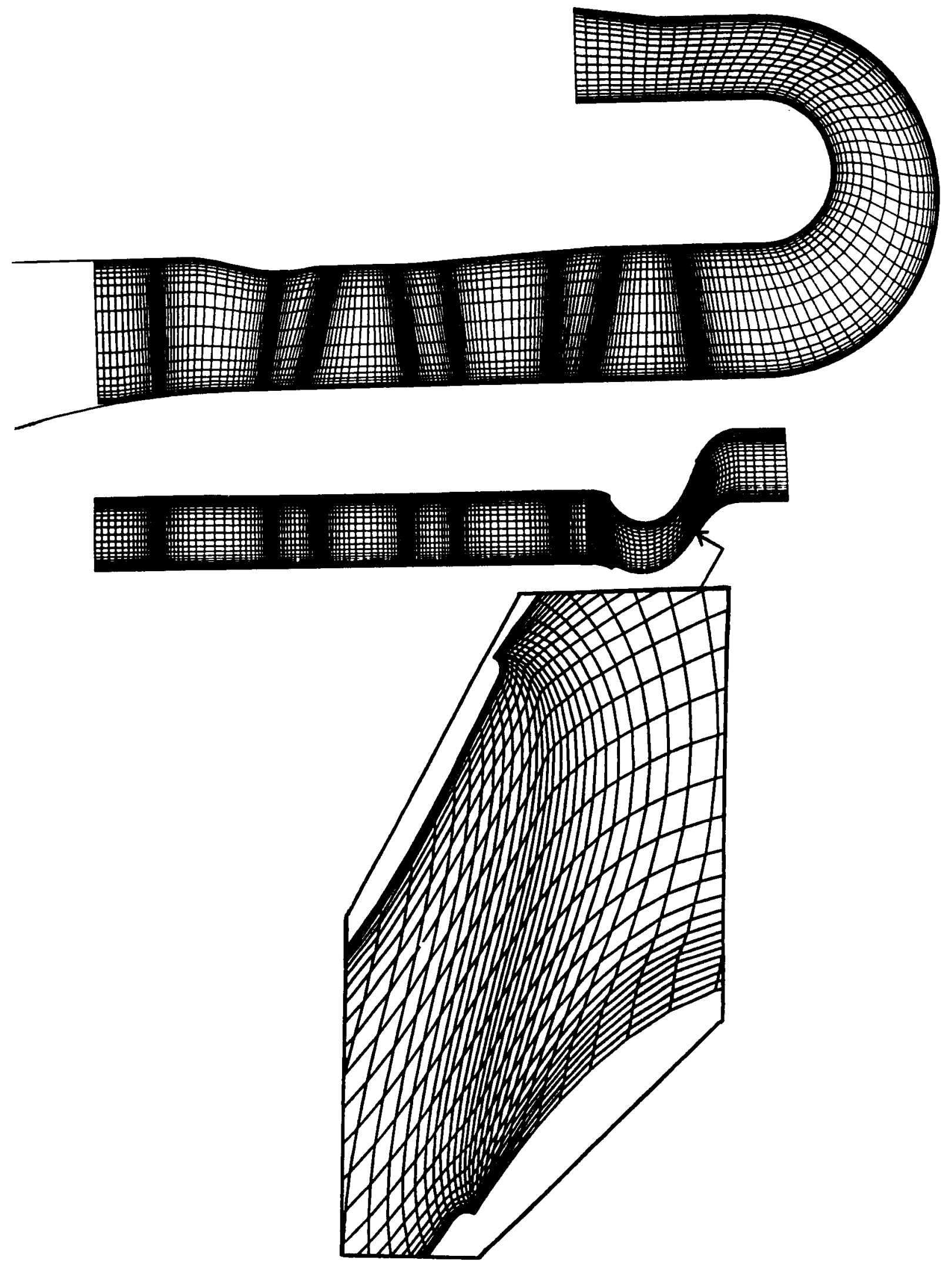

Figure 6. The top plot is an axisymmetric view of the full turbopump flowpath. The leading and trailing edges of each blade are evident due to grid clustering. Below that is a blade-to-blade grid for the last blade row, where the first three blades will be represented by body forces. An enlargement of the trailing edge of the fourth blade is shown in the unset. 


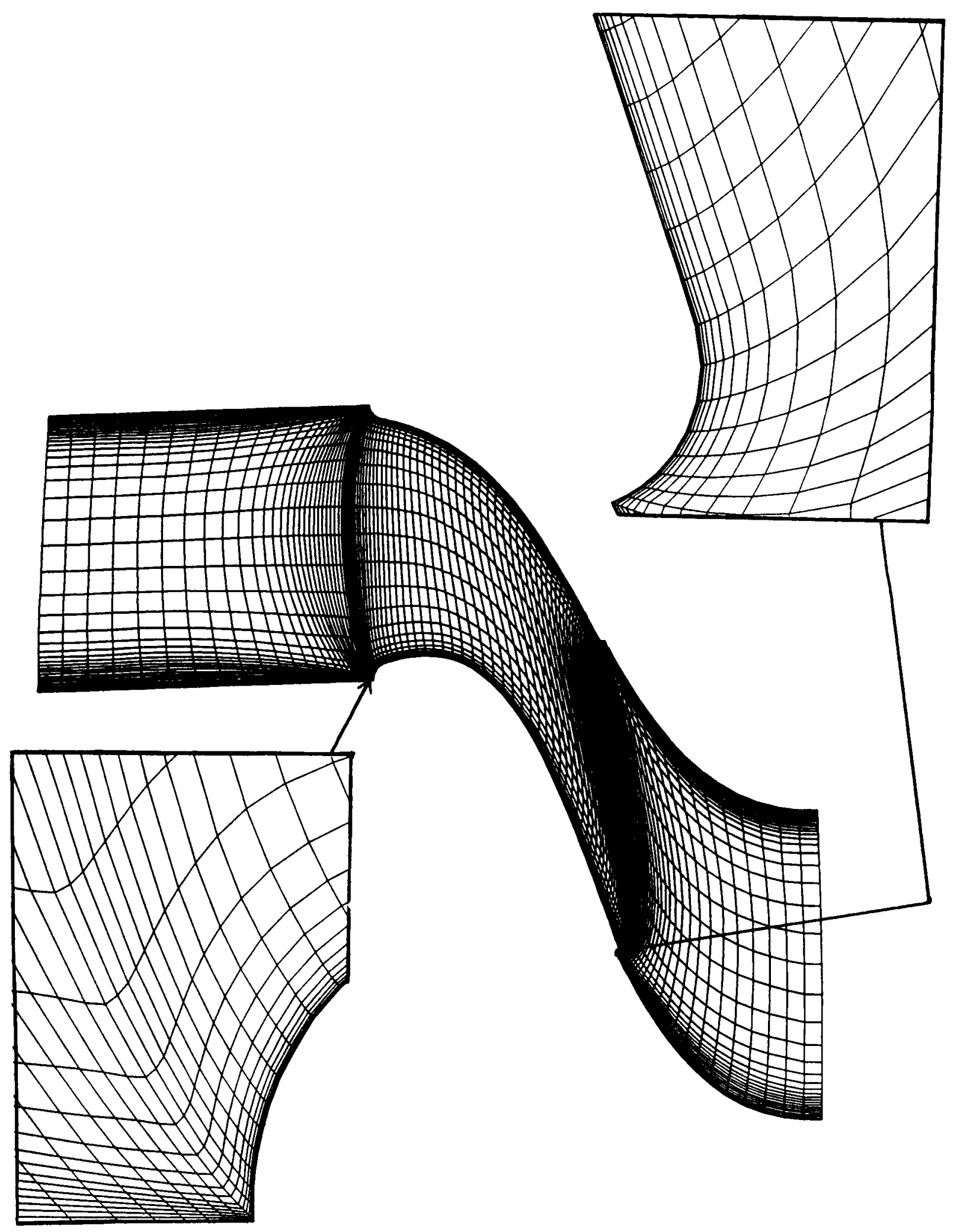

Figure 7. A blade-to-blade view of the grid at mid-span of the first stator of the UTRC large scale rotating rig. The insets show enlargements of the leading and trailing edges. This grid is $121 \times 41 \times 41$. 

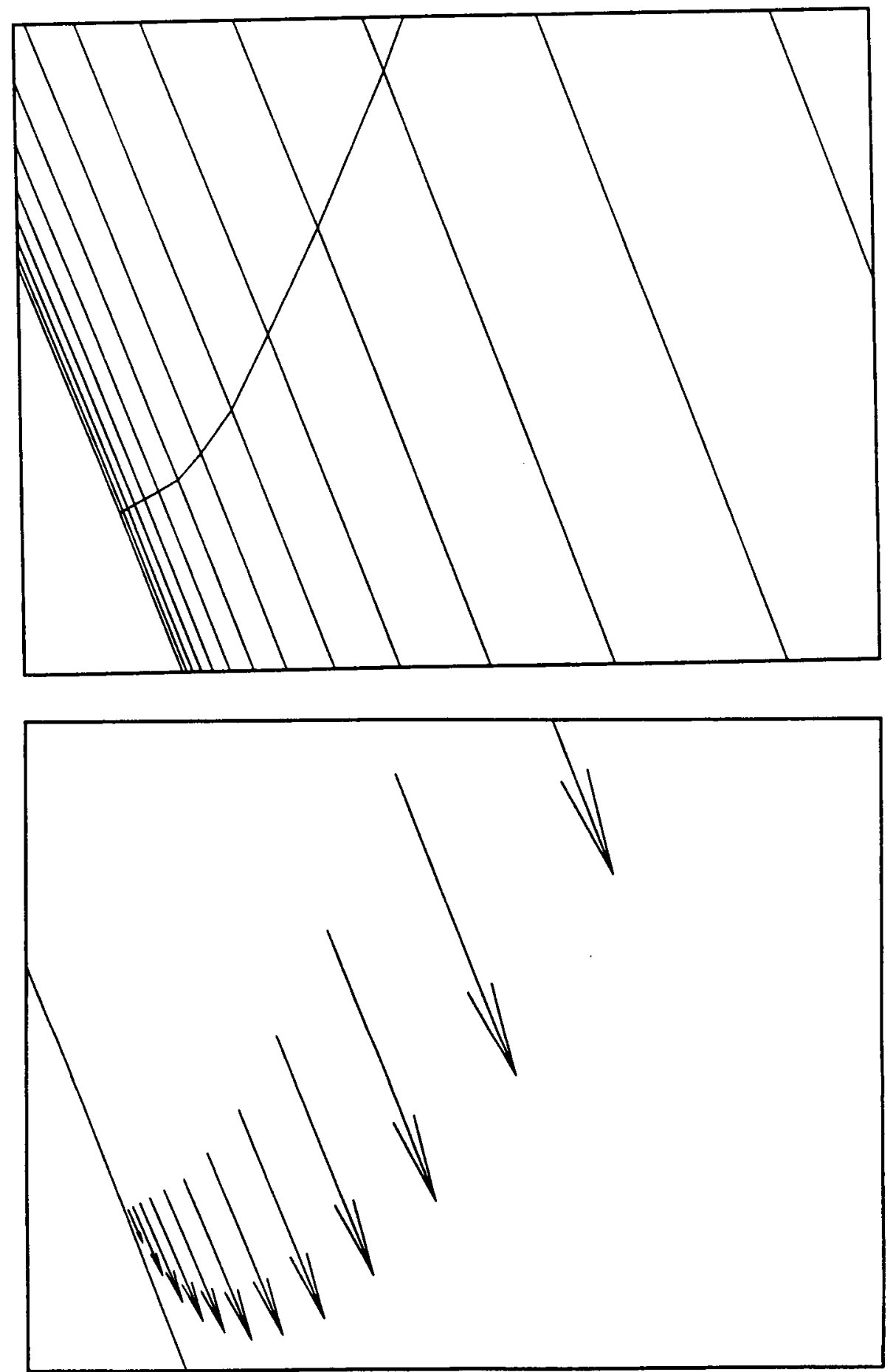

Figure 8. An enlargement of a surface point on the suction side near the trailing edge. The grid and vector plots from a Navier Stokes solution are plotted. 


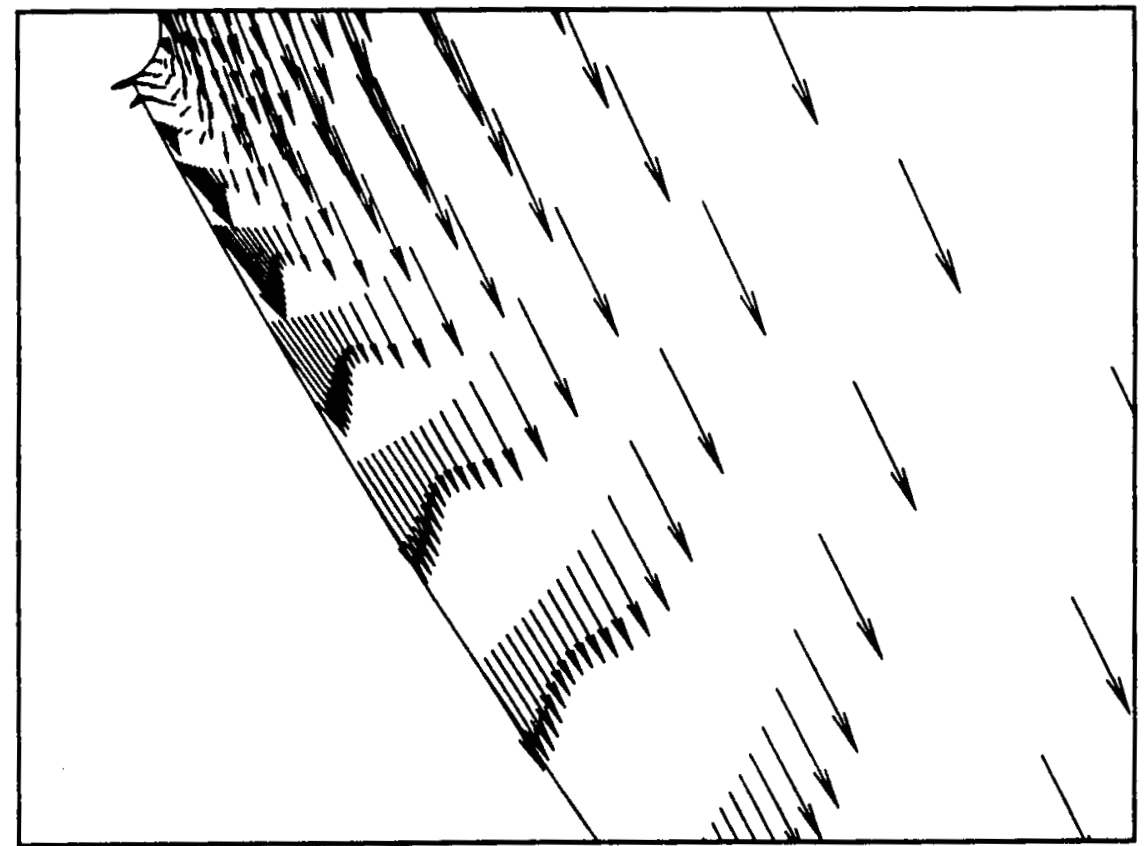

Figure 9. Velocity vectors indicating the separation bubble and wake at mid-span of the UTRC large scale rotating rig. Only vectors on alternate axial grid lines are plotted.

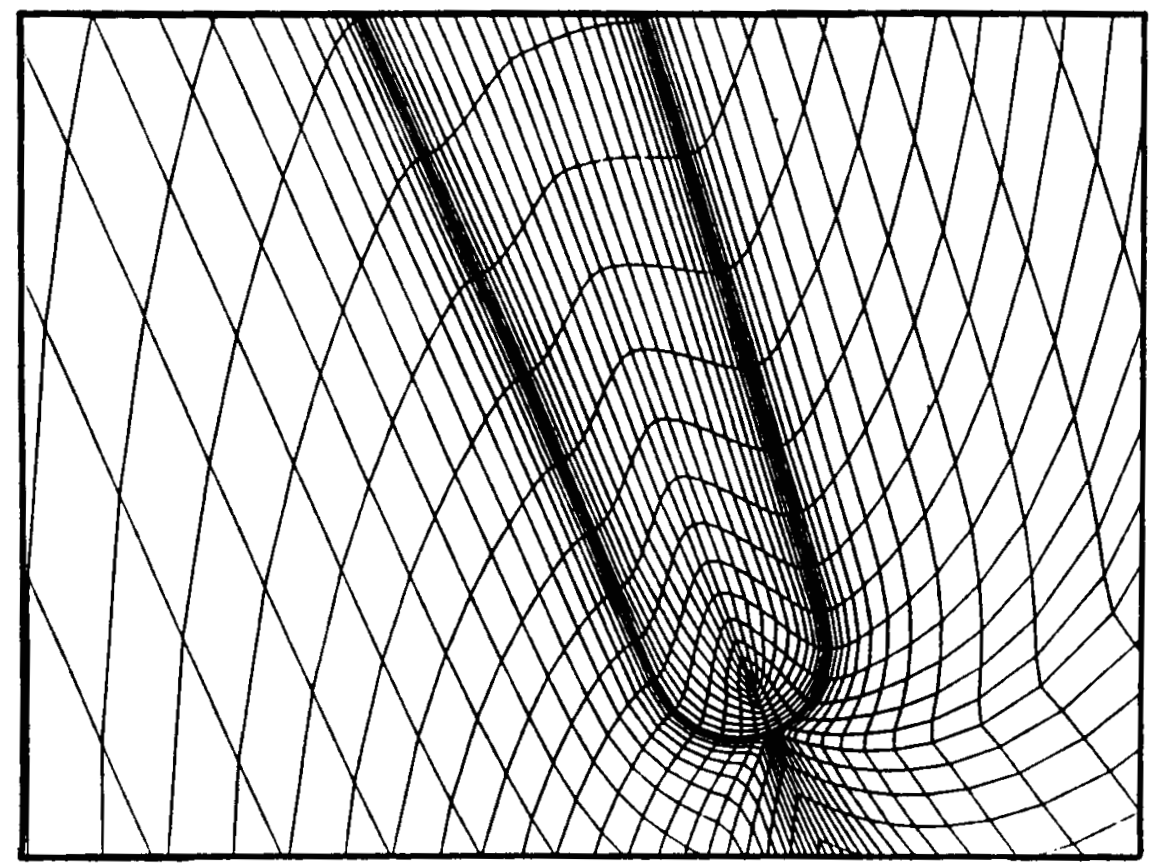

Figure 10. Tip clearance grid for a typical turbine blade shown along with the blade to blade grid. 


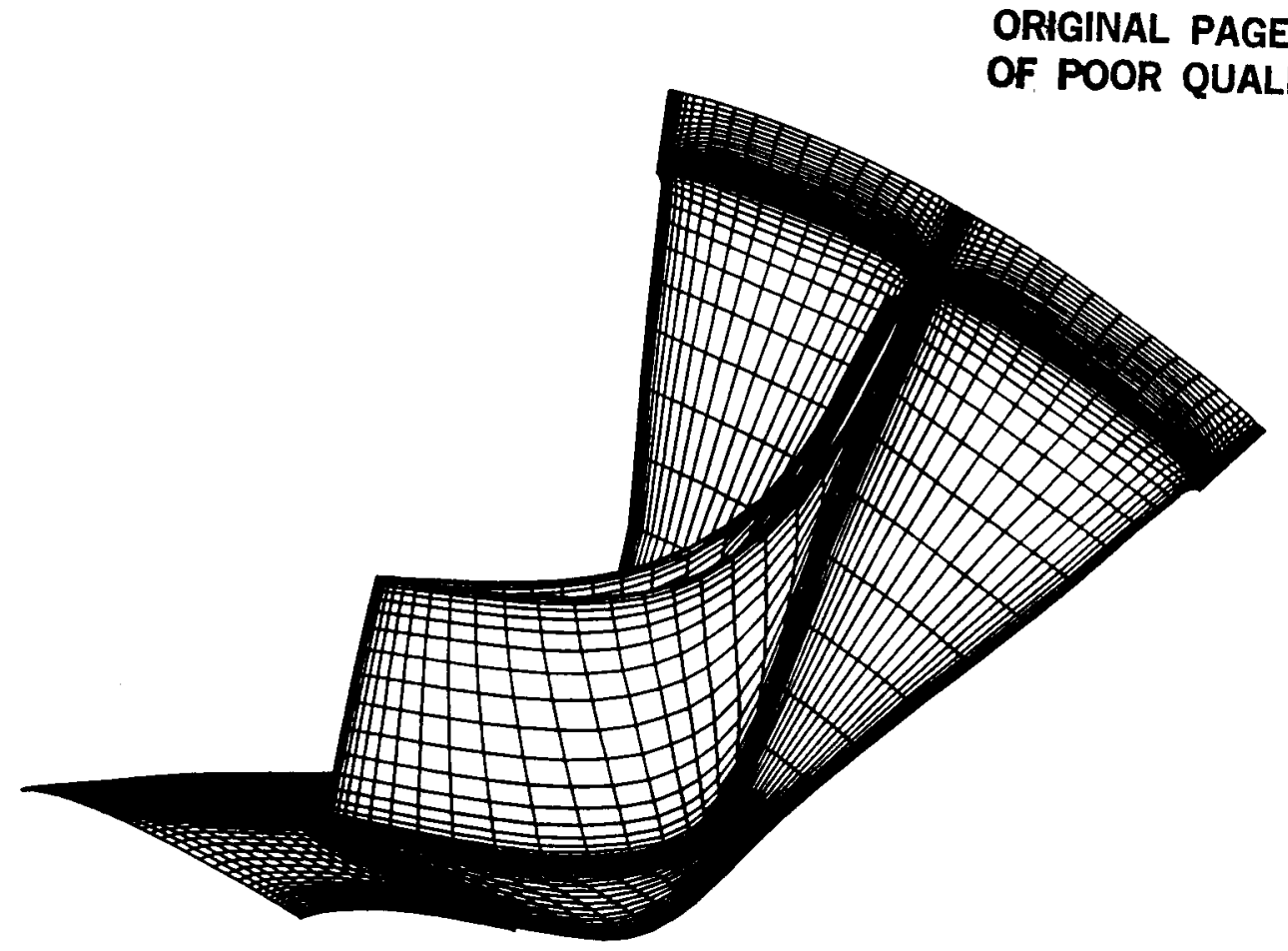

Figure 11. A perspective view of a radial inflow turbine grid showing the blade and hub surfaces.

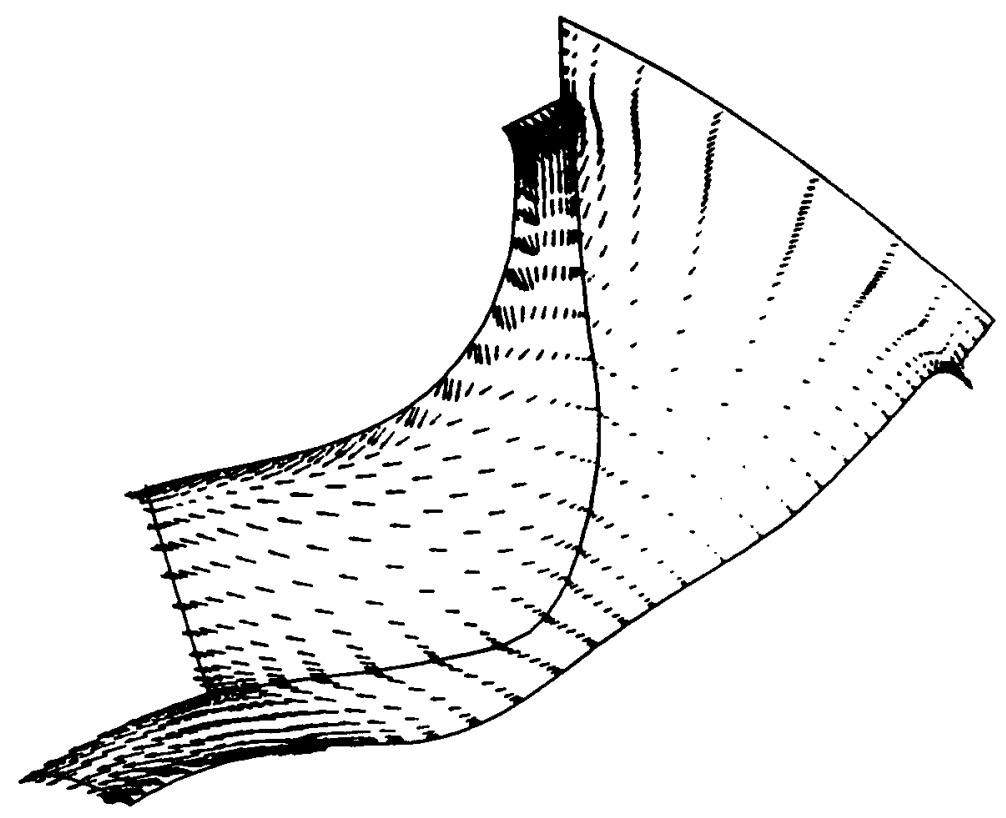

Figure 12. Velocity vectors plotted adjacent to the hub and blade suction surfaces for the radial inflow turbine. 


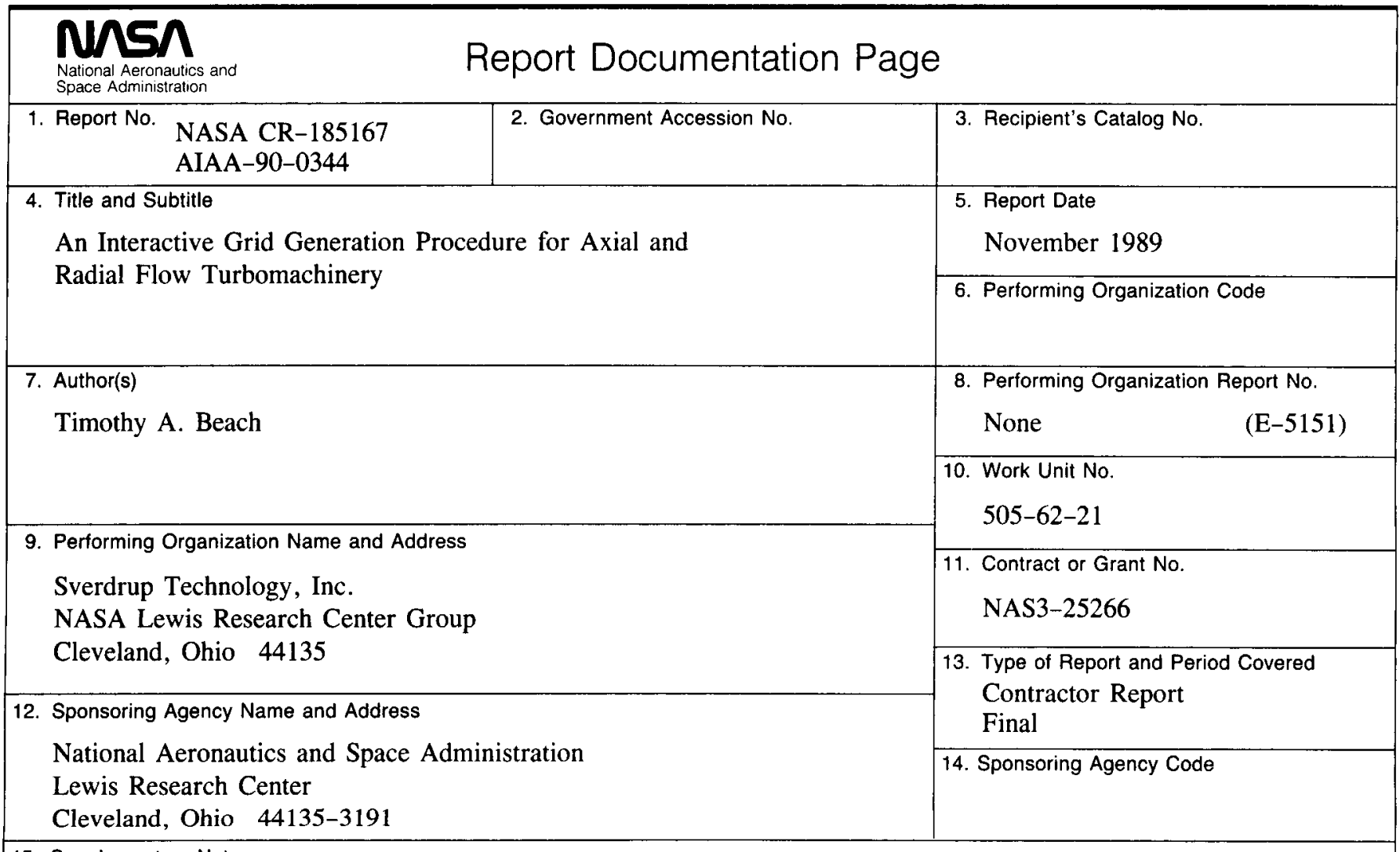

15. Supplementary Notes

Project Manager, John J. Adamczyk, Office of Chief Scientist, NASA Lewis Research Center. Prepared for the 28th Aerospace Sciences Meeting sponsored by the American Institute of Aeronautics and Astronautics, Reno, Nevada, January 8-11, 1990.

\section{Abstract}

A combination algebraic/elliptic technique is presented for the generation of three dimensional grids about turbomachinery blade rows for both axial and radial flow machinery. The technique is built around use of an advanced engineering workstation to construct several two dimensional grids interactively on predetermined blade-to-blade surfaces. A three dimensional grid is generated by interpolating these surface grids onto an axisymmetric grid. On each blade-to-blade surface, a grid is created using algebraic techniques near the blade to control orthogonality within the boundary layer region and elliptic techniques in the mid-passage to achieve smoothness. The interactive definition of bezier curves as internal boundaries is the key to simple construction. This procedure lends itself well to zonal grid construction, an important example being the tip clearance region. Calculations done to date include a space shuttle main engine turbopump blade, a radial inflow turbine blade, and the first stator of the United Technologies Research Center large scale rotating rig. A finite volume Navier Stokes solver was used in each case.

17. Key Words (Suggested by Author(s))

Grid generation

Turbomachinery
18. Distribution Statement

Unclassified - Unlimited

Subject Category 61 